\title{
Editorial
}

Andreas Fischer*

\section{Nichtinvasive Messverfahren für Mikro- und Makroströmungen}

\author{
Non-invasive measurement techniques for micro- and large-scale flows
}

https://doi.org/10.1515/teme-2022-0019

Eingang 25. Januar 2022; angenommen 25. Januar 2022

Liebe Leserinnen und Leser,

Strömungen sind allgegenwärtig, beispielsweise beim Atmen, bei der Ausbreitung von Partikeln in einem Raum, beim Zufügen von Milch in den Kaffee, beim Abführen von Wärme und bei der Wandlung von Windenergie in elektrisch nutzbare Energie. Deshalb verwundert es nicht, dass sowohl international als auch national die Strömungsforschung ein lebendiges, hochaktuelles Fachgebiet darstellt.

Vom 7. bis zum 9. September 2021 fand an der Universität Bremen die 28. Fachtagung „Experimentelle Strömungsmechanik“ der Deutschen Gesellschaft für LaserAnemometrie GALA e. V. statt. Hierbei hat das Bremer Institut für Messtechnik, Automatisierung und Qualitätswissenschaft (BIMAQ) mit großer Freude die Organisation dieser Präsenzveranstaltung angenommen. Nach einer pandemiebedingter Pause im Vorjahr, wurde die Fachtung wieder zu einem lebendigen Ort des fachlichen Austauschs über den aktuellen Fortschritt in der experimentellen Strömungsforschung.

Ein zentrales Thema in den insgesamt 52 Tagungsbeiträgen waren Strömungsmessprinzipien und deren Anwendung in Experimenten, um strömungsmechanische Fragestellung beantworten zu können. Deshalb, und wegen der großen Resonanz der Teilnehmerinnen und Teilnehmer, lag es nahe, mit einem Schwerpunktheft Einblicke in ausgewählten Forschungsthemen $\mathrm{zu}$ „Nichtinvasiven Messverfahren für Mikro- und Makroströmungen“ zu ermöglichen. Mit den nachfolgende Themen wird ein Ausschnitt aus der großen Vielfalt berührungsloser Messprinzipien (Magnetresonanztomographie, Partikelbild-Velozimetrie, Laser-Doppler-Velozi-

*Korrespondenzautor: Andreas Fischer, Universität Bremen, Bremer Institut für Messtechnik, Automatisierung und Qualitätswissenschaft (BIMAQ), Linzer Str. 13, 28359 Bremen, Germany, E-Mail: andreas.fischer@bimaq.de, ORCID:

https://orcid.org/0000-0001-7349-7722 metrie), die Messung von sowohl Strömungsgeschwindigkeiten als auch -temperaturen, und verschiedene Anwendungsfelder vorgestellt. Als Gastherausgeber danke ich allen Autoren für ihre Bereitschaft, Ihnen - liebe Leserin oder lieber Leser - einen spannenden Einblick in aktuelle Forschungsarbeiten zur Strömungsmesstechnik zu ermöglichen.

Sollte nach diesen Einblicken der Wunsch nach einer Ausweitung und Vertiefung der Materie entstehen, so sei an dieser Stelle ein Blick in den Tagungband empfohlen [1]. Zunächst aber, wünsche ich Ihnen viel Vergnügen beim Lesen der Beiträge des vorliegenden Schwerpunktheftes!

Ihr

Andreas Fischer

\section{Literatur}

1. A. Fischer, D. Stöbener, C. Vanselow, B. Ruck, and A. Leder, editors. Experimentelle Strömungsmechanik - 28. Fachtagung. Deutsche Gesellschaft für Laser-Anemometrie - German Association for Laser Anemometry GALA e. V., Karlsruhe, 2021.

\section{Autoreninformationen}

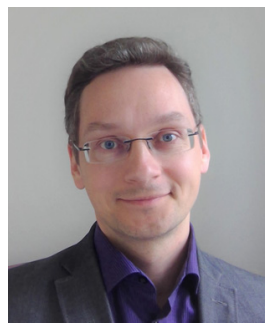

\section{Andreas Fischer}

Universität Bremen, Bremer Institut für Messtechnik, Automatisierung und Qualitätswissenschaft (BIMAQ), Linzer Str. 13, 28359 Bremen, Germany andreas.fischer@bimaq.de

Andreas Fischer leitet seit 2016 das Fachgebiet Messtechnik, Automatisierung und Qualitätswissenschaft im Fachbereich Produktionstechnik der Universität Bremen. Seine Hauptarbeitsfelder sind die Untersuchung und Anwendung optischer Messsysteme für die Analyse von Strömungs- und Fertigungsprozessen an den Grenzen der Messbarkeit. Für seine Dissertation erhielt er mehrere Auszeichnungen, darunter den AHMT-Messtechnikpreis 2010. 